Del Río CABrera, Juan Antonio: Texto y contexto. Los géneros del folklore oral en Olvera y Alpandeire. UNED, 2015, $879 \mathrm{p}$.

\title{
Texto y contexto. Los géneros del folklore oral en Olvera y Alpandeire
}

David MAÑERo LOZANO

Universidad de Jaén

No es una práctica muy frecuente reseñar tesis doctorales, pero el alcance e interés de la publicada por Del Río Cabrera justifica esta excepción. La obra, publicada en formato de e-book con bastante posterioridad a la fecha de defensa de la tesis, ${ }^{\mathrm{I}}$ arranca con algunas disquisiciones sobre la pertinencia de la terminología empleada en los estudios sobre folclore, a la que el autor atribuye una escasa uniformidad y consistencia teórica. En particular, se incide en la supuesta inexactitud de denominaciones como literatura oral o textos orales, dado que «apuntalan el supuesto isomorfismo, más que discutible, existente entre las tradiciones orales y las literarias» (p. I3) y que «Los ítems folklóricos orales no son textos, sino fenómenos inseparables de su producción y, por tanto, de sus contextos de uso» (p. I7). Se trata de apreciaciones que, sin duda, ponen de manifiesto una carencia metodológica especialmente perceptible en las investigaciones folclóricas del ámbito hispánico peninsular, en las que el estudio de las manifestaciones orales se ha restringido a un enfoque fundamentalmente filológico.

En efecto, la denominada «escuela filológica española», que durante décadas canalizó la mayor parte de las investigaciones sobre los testimonios orales, priorizó el estudio de los elementos textuales e historiográficos en detrimento de los aspectos relacionados con los contextos de producción. Unido a esto, su monumental empresa de recuperación del patrimonio oral se restringió a determinadas manifestaciones, como el romancero patrimonial, que gozaban del prestigio de una larga tradición, con antecedentes documentados en las fuentes impresas o manuscritas más antiguas. Hemos perdido, por tanto, la oportunidad de conocer las últimas muestras de otras muchas formas de expresión orales, además de una valiosa información sobre los condicionantes culturales que propiciaron su difusión.

Sin embargo, el hecho de que la oralidad se desarrolle necesariamente dentro de un contexto, que condiciona tanto su configuración formal como su alcance temático e intencional, no implica que las manifestaciones orales carezcan de una dimensión textual, naturalmente diversa de los textos producidos y transmitidos por escrito. En este sentido, la propia escuela filológica acuñó la noción de apertura textual para referirse al carácter específico de los textos orales, que de ningún modo implica ese «isomorfismo» o equiparación entre tradiciones orales y escritas criticado por Del Río Cabrera. De este modo, en la base de los planteamientos suscritos por este autor, subyace también una postura metodológica restrictiva. Su reivindicación de los aspectos relacionados con la ocasionalidad, tan apremiante como legítima, se presenta innecesariamente como una alternativa a las investigaciones de índole textual. Oralidad, escritura y contexto mantienen, a mi jui-

I Disponible en versión Kindle en <https://www.amazon.es/contexto-g\%C3\%Agnerosfolklore-Olvera-Alpandeire-ebook/dp/Bo7D56PV58> [fecha de consulta: octubre de 2020]

Estudis de Literatura Oral Popular, núm. 9, 2020, I67-I7I | DOI: I0.I7345/elop2020I67-I7I ISSN: 2OI4-7996 | http://revistes.urv.cat/index.php/elop 
cio, relaciones de dependencia que legitiman las investigaciones llevadas a cabo desde los distintos ámbitos de la investigación, cuando no desde una perspectiva integradora. El empleo, por tanto, de los membretes literatura oral y textos orales responde a la necesidad de describir un tipo de manifestaciones diferenciadas respecto de las producciones escritas no oralizadas, es decir, una forma de expresión en la que la dimensión textual, incluidos aquellos elementos relacionados con la escritura, se halla asociada ineludiblemente a un contexto de transmisión oral.

El autor acomete seguidamente una revisión crítica de las propuestas clasificatorias de los géneros folclóricos. En términos generales, propone una enmienda a la totalidad de las aportaciones teóricas más difundidas, fundamentalmente las impulsadas por la escuela finesa y sus desarrollos, a los que reprocha la falta de atención a los factores contextuales. No existen motivos, sin embargo, con independencia del valor que le atribuyamos a las circunstancias concretas de la transmisión oral, para negar la utilidad y el poder descriptivo del sistema clasificatorio de motivos y tipos folclóricos, al que el propio autor recurre ocasionalmente. A pesar de las múltiples incoherencias, imprecisiones y lagunas de este método, señaladas incluso por sus representantes, la clasificación en tipos folclóricos sigue aportando caracterizaciones tipológicas y análisis textuales relevantes y perfectamente compatibles con los estudios basados en los factores culturales que propician el desarrollo concreto de las manifestaciones orales. No es posible ni conveniente, claro está, reducir a esquemas clasificatorios muchas de las muestras de la tradición, ni procede tampoco considerar cerrada una investigación en la fase de descripción tipológica, pero estas circunstancias, presentes en cualquier disciplina de estudio, no ofrecen argumentos para deslegitimar las investigaciones tipológicas.

Hechas estas disquisiciones preliminares, que requerirían de otros muchos argumentos y ejemplos que no es posible exponer en el espacio de una reseña, se entenderán mejor mis impresiones sobre la obra. Al margen del enfoque reduccionista al que acabo de referirme, en mi opinión prescindible, considero que son muchas y muy valiosas las aportaciones que contiene el libro de Del Río Cabrera.

Destaca, en primer lugar, su ágil y documentado esfuerzo de descripción de las principales aportaciones teóricas al estudio del folclore, además del amplio estado de la cuestión sobre las labores de recopilación etnográfica llevadas a cabo en Andalucía. A lo largo de los apartados introductorios y otros epígrafes posteriores, se desbroza un complejo panorama crítico en el que se maneja un volumen de publicaciones amplio y representativo cuyas aportaciones se sintetizan y contrastan eficazmente. Unido a esto, mediante el recurso a comunicaciones personales realizadas por investigadores con los que el autor mantuvo correspondencia, como Ben-Amos, se intercalan algunas matizaciones teóricas a los aspectos reseñados en el estado de la cuestión. Me abstengo, en cualquier caso, de comentar las frecuentes apreciaciones subjetivas introducidas por el autor, en mi opinión prescindibles en el marco de un trabajo académico.

Otro aspecto positivo es el escrúpulo metodológico apreciable, por ejemplo, en los comentarios acerca de las categorizaciones genéricas nativas (apartado I.2.2), en los que se conjuga la observación directa de la historicidad de los géneros con la mediación operada por el contexto de transmisión y la influencia de la cultura escrita. Apreciaciones de este tipo son fundamentales, según creo, tanto 
durante el proceso de documentación de materiales etnográficos como en la fase de clasificación e interpretación de los repertorios orales.

La investigación desarrollada por Del Río Cabrera parte del trabajo de campo realizado fundamentalmente en Andalucía, donde prestó una mayor atención a las comarcas de la Costa del Sol occidental (Málaga), al Campo de Gibraltar (Cádiz) y a las sierras que limitan las provincias de Cádiz, Málaga y Sevilla, con particular atención a las localidades de Olvera y Alpandeire.

A lo largo de los apartados dedicados a la descripción histórica y sociocultural de estas poblaciones, el autor va desgranando con rigor y minuciosidad una vasta información procedente de archivos y otras fuentes especializadas, además de intercalar con pertinencia los testimonios orales recopilados (letras carnavalescas, relatos sobre aparecidos, leyendas fundacionales, anécdotas sobre bandoleros, dichos locales, etc.). Se echa en falta, sin embargo, que este rico repertorio esté accesible, junto al resto de muestras registradas por el autor, en apartados específicos dedicados a cada tipo de manifestación oral o, en su defecto, que se anexe un índice que facilite la localización de los registros dispersos en los epígrafes que se consagran a la historia y a las particularidades de la sociedad y la cultura de Olvera y Alpandeire. La dimensión textual enunciada en el primer término del título de la obra (Texto y Contexto) queda, por tanto, en un plano marginal (conscientemente marginado) tanto por las dificultades de consulta directa de los testimonios como por el hecho de que se ofrezcan tan solo aquellas muestras seleccionadas para ilustrar aspectos relativos al contexto. El afán de priorizar esta labor de supuesta reconstrucción del contexto queda además patente en los esfuerzos dedicados a algunas manifestaciones del folclore material, como es el caso de la arquitectura popular, con lo que se amplían las aportaciones del libro más allá del propósito enunciado en el subtítulo de estudiar «los géneros del folklore oral».

Con estas observaciones no pretendo restar importancia al estudio del contexto ni relativizar el alcance de sus resultados. Comparto, de hecho, la opinión de Del Río Cabrera al afirmar que la «recuperación de manifestaciones de distintos géneros, que muchos recopiladores se marcan como su finalidad principal, no puede realizarse mediante colecciones inventariadas de materiales descontextualizados y desenraizados de las personas que los usan» (p. 242). Sin embargo, la conveniencia de recabar los máximos datos posibles sobre los informantes y las circunstancias de la transmisión oral no justifica, según creo, el «interés por desligar este trabajo, y otros que ya hemos realizado, de las labores de recopilación folklórica, tan comunes aún en España», que, en palabras del autor, «tiene que ver más con la pobreza de sus resultados teóricos» (p. 242).

Llegados a este punto, que en mi opinión implica una reducción al absurdo de las relaciones entre el trabajo de documentación y la labor interpretativa, cabría incluso objetar que este estudio del contexto, en la medida en que se desliga de la labor de recopilación, no es propiamente una investigación sobre el contexto, en el sentido de que este es un término relacional que presupone la conexión con los datos que supuestamente se contextualizan. En efecto, lo que realmente conduce a esa pobreza de resultados teóricos a la que alude Del Río Cabrera no es, según creo, la documentación de repertorios orales por parte de otros investigadores, sino la decisión que el autor toma de restringir el recurso a las manifestaciones orales en aquellos casos en los que, puntualmente, estas le permiten ilustrar determinados aspectos de la sociedad o cultura estudiada. 
Del Río Cabrera no se propone, por tanto, el objetivo de recopilar una muestra rigurosa y sistemática de los «ítems folklóricos orales» (términos que emplea para eludir la denominación de «textos orales» o de «etnotextos»). Su metodología consiste, según afirma, en «recoger las expresiones en las situaciones cotidianas, el llamado contexto natural o contexto de uso, sin recurrir a medios evidentes de recogida, como el consabido cassette o el inocente bolígrafo, que son a la vez eficaces mecanismos de distorsión» (p. 240). El recurso a las grabaciones de vídeo se considera aún más pernicioso: «si se usa el vídeo es todavía bastante peor» (p. 22I). Con todo, después de estas consideraciones, el autor apostilla, como mal menor: «Cuando la memoria, o las notas apresuradas que tomábamos inmediatamente después, no nos acompañaban debido a la amplitud del ítem, realizábamos grabaciones para recogerlos» (p. 240); y, en efecto, a lo largo del trabajo se menciona que determinados registros fueron grabados. El autor no precisa, en cualquier caso, en qué momentos empleó su memoria.

Me parece conveniente ejemplificar también el procedimiento de trabajo desarrollado como alternativa a las «hipótesis y métodos desfasados» (p. 242) que a su juicio han conducido las labores de recopilación folclórica mediante las técnicas de encuesta y grabación convencionales. Como ejemplo de esto, me referiré al extenso apartado denominado «5.3. La mañana» (p. 289-335). En este, el autor acude como estrategia o esquema retórico a la descripción de una especie de recorrido por las calles desde primera hora del día hasta el momento de comer. No se trata de una mañana concreta, sino de una franja del día en la que habitualmente obtuvo información sobre diversas manifestaciones orales. Encontramos, de este modo, una sucesión imprevista de párrafos dedicados a describir y comentar el uso de algunos refranes, saludos, apodos, puyas y chistes que el autor escuchó durante sus recorridos matutinos. Los testimonios aludidos, así como los comentarios que estos suscitan, revierten un indudable interés, si bien se echan en falta algunos datos fundamentales sobre los transmisores, a los que no se identifica: «Dice un hombre...» (p. 294), «otro de Bobadilla proclama que...» (p. 294), «dice otra un refrán que también hemos oído a algunos hombres...» (p. 295), «Nos lo contó una viuda» (p. 297), «nos contó el que supuestamente era su tataranieto...» (p. 3II), «una muchacha» (p. 3I6), «un octogenario al que le gusta mucho hablar con la gente» (p. 328), etc. Encontramos también casos en los que tampoco se indica la fecha de recopilación. El autor se limita a recordar «Otra versión olvereña» (p. 295) o a referir lo que «dice uno de los asistentes...» (p. 296) o la contestación de «un hombre cercano a los setenta años, al ser preguntado por el refrán que más utiliza» (p. 296).

Como ya comenté anteriormente, sería de interés que este tipo de testimonios, cuya ubicación no es previsible dentro del mencionado epígrafe, se identificasen en un índice, que a su vez ofreciese los datos de los informantes, por no mencionar la conveniencia de aportar una descripción biográfica de los transmisores y de especificar los criterios empleados en el registro y la transcripción de las muestras orales. Se trata de prácticas etnográficas perfectamente compatibles con el método de estudio empleado, a las que, en mi opinión, debe recurrirse para profundizar en los aspectos relativos al contexto y los transmisores, además de garantizar la fiabilidad de los testimonios aportados.

Se ofrecen seguidamente los apartados titulados «5.4. La tarde» y «5.5. La noche», además de otra serie de epígrafes sobre los «contextos formales» correspon- 
dientes a las distintas festividades. Los siguientes capítulos, que reflejan la amplitud e interés del enfoque adoptado en la investigación, se centran en aspectos tan variados y pertinentes desde el punto de vista folclórico como las circunstancias históricas, la división «de género», la estratificación social, las minorías (ingleses, marroquíes y gitanos) y las identidades locales. Mediante un procedimiento análogo al empleado en el apartado «La mañana», muchas de las muestras orales, como los villancicos o canciones de carnaval, por mencionar un par de ejemplos, se transcriben sin identificar a los transmisores ni indicar tampoco la fecha de registro, informaciones que el autor solo evita omitir al referirse a fuentes transmitidas por escrito (v. gr.: «en la misma página, recoge una letrilla procedente de un artículo del Sur de Málaga escrito por Juan Antonio Morgado el I3 de enero de I985", 442-443).

Se exponen a continuación las conclusiones y los posibles desarrollos futuros de las investigaciones más recientes del autor, que se desgranan a lo largo de casi un centenar de páginas, en múltiples apartados que abarcan cuestiones muy variadas, desde asuntos concretos, como los aniversarios marianos en Olvera o la apertura de la casa natal de fray Leopoldo en Alpandeire, hasta aspectos teóricos de primer relieve, como la performance, los contextos, las situaciones, los temas, los métodos de recogida o los géneros, entre otros.

La obra se cierra con un voluminoso apartado de fuentes (pp. 745-827) que da la medida del enorme caudal de documentación y estudios empleados. El repertorio manejado presenta tales dimensiones que el lector habrá de excusar la omisión en el listado de algunos repertorios orales importantes con muestras andaluzas, como los de Fraile Gil, e incluso de referencias que están citadas en el cuerpo del trabajo, como la de Pedrosa (I995), que supongo que alude al artículo titulado «El repertorio romancístico de una mujer de Puentegenil (Córdoba)» publicado ese año en el número I76 de la Revista de Folklore. O tal vez se refiera a la consulta del Corpus de Literatura Oral (2015-), plataforma que, entre sus casi 3500 registros procedentes de Andalucía, contiene parte de las grabaciones de audio y transcripciones de las muestras orales mencionadas. Se anexa, por último, un apéndice documental con planos de las zonas estudiadas y treinta y seis testimonios fotográficos de Olvera, Alpandeire y otros lugares cuya relación con el libro tal vez debiera explicitarse; como la última fotografía, a la que acompaña el lema «Campo de concentración de Buchenwald situado junto a Weimar, la capital cultural de Alemania» (p. 879). 